\title{
FÁCIL E RÁPIDA OBTENÇÃO DA CELULOSE NANOCRISTALINA MAGNÉTICA ${ }^{1}$
}

\author{
EASY AND FAST OBTAINING OF \\ MAGNETIC NANOCRYSTALLINE CELLULOSE
}

\section{Franciele da Silva Bruckmann'2, Theodoro da Rosa Salles ${ }^{3}$, Magna Tainar Walczak Reimann ${ }^{3}$, Liana da Silva Fernandes ${ }^{4}$, Ivana Zanella da Silva ${ }^{4}$, Sergio Roberto Mortari ${ }^{4}$ e Cristiano Rodrigo Bohn Rhoden ${ }^{5}$}

\section{RESUMO}

Nos últimos anos os polímeros têm se mostrado muito versáteis e, em virtude disso, inúmeros trabalhos na área científica vêm sendo realizados. A nanocelulose é um biopolímero de origem natural, obtida através de diferentes técnicas, sejam estas químicas, físicas ou biológicas. Entretanto, a literatura mostra-se deficiente de trabalhos que reportam a sua incorporação a nanopartículas magnéticas. Neste sentido, este trabalho teve como objetivo a obtenção fácil e rápida da celulose nanocristalina magnética, para posteriormente utilizá-la em diferentes aplicações, sejam estas tecnológicas ou biológicas. O uso desta metodologia mostrou-se eficaz na obtenção da celulose nanocristalina decorada com diferentes quantidades de ferrita $\left(\mathrm{CNC}\right.$. $\left.\mathrm{Fe}_{3} \mathrm{O}_{4}\right)$. Por meio das caracterizações foi possível verificar a presença da ferrita nas estruturas, bem como, constatar que as condições experimentais contribuíram com a diminuição do tamanho da partícula e do grau de cristalinidade das amostras.

Palavras-Chave: Bionanopolímero, Magnetização, Nanopartículas magnéticas.

\section{ABSTRACT}

In recent years, polymers have proved to be very versatile material. As a result, numerous works in the scientific area have been reported. Nanocellulose is a natural biopolymer, obtained through different techniques, whether chemical, physical, or biological. However, the literature shows a lack of studies that report its incorporation to magnetic nanoparticles. In this sense, this work objective the easy and fast obtention of magnetic nanocrystalline cellulose, to apply in different applications, technological as well as biological. The methodology proved to be effective in obtaining nanocrystalline cellulose decorated with different amounts of ferrite $\left(\mathrm{CNC}^{\mathrm{F}} \mathrm{Fe}_{3} \mathrm{O}_{4}\right)$. Through the characterizations it was possible to verify the presence of ferrite in the structures, as well as, to verify that the experimental conditions contributed to the decrease of the particle size and the degree of crystallinity of the samples.

Keywords: Bionanopolymer, Magnetization, Magnetic nanoparticles.

\footnotetext{
${ }^{1}$ Trabalho de Pesquisa.

${ }^{2}$ Mestranda - Programa de Pós-Graduação em Nanociências - Universidade Franciscana. E-mail: francielebruckmann2@ gmail.com

${ }^{3}$ Acadêmicos do curso de Engenharia Química - Universidade Franciscana. E-mails: theodoro.rsalles@gmail.com; magna.walre@gmail.com

${ }^{4}$ Colaboradores. Professores do curso de Engenharia Química e Programa de Pós-Graduação em Nanociências - Universidade Franciscana. E-mail: liafernandesqmc@gmail.com; ivanazanella@gmail.com; smortari@hotmail.com

${ }^{5}$ Orientador. Professor do curso de Engenharia Química e Programa de Pós-Graduação em Nanociências - Universidade Franciscana. E-mail: cristianorbr@gmail.com
} 


\section{INTRODUÇÃO}

Os polímeros têm atraído grande interesse na pesquisa científica, pois muitos caracterizam-se por serem biodegradáveis, biocompatíveis e com baixa toxicidade (ZINGE; KANDASUBRAMANIAN, 2020). A celulose é um homopolímero linear de $\beta$ - $D$-glicose unido pela ligação 1,4-glicosídica, é um dos biopolímeros de maior ocorrência no meio ambiente, sendo constituinte principal da parede celular de plantas (ZAMAN et al., 2020). A nanocelulose (NC) é um nanomaterial derivado da celulose, obtida por meio de processos químicos, oxidativos (SALIMI et al., 2019), enzimáticos (RIBEIRO et al., 2019) e biológicos (STANISŁAWSKA, 2016).

No campo da nanotecnologia, tanto os derivados alótropos de carbono quanto os polímeros de origem natural, em função de suas aplicações, demonstrando serem um campo de estudo em grande expansão (VIANA et al., 2019).

A nanocelulose apresenta características excepcionais como elevada área superficial, alta resistência e dispersividade (TAN; OOI; LEO, 2020). Além disso, dispõe de grupos funcionais oxigenados, conferindo-lhe a capacidade de modificação química e formação de nanocompósitos (ZINGE; KANDASUBRAMANIAN, 2020). A NC pode ser dividida em três categorias: celulose nanocristalina, nanofibras de celulose e celulose bacteriana, essa diferenciação decorre dos métodos de síntese, tamanho e morfologia (LIN; SHATKIN; KONG, 2019; THOMAS et al., 2020).

Recentemente a literatura têm reportado o emprego de nanocompósitos contendo $\mathrm{NC}$ em estudos de atividade fotocatalítica (LEFATSHE; MUIVA; KEBAABETSWE, 2017), desenvolvimento de embalagens alimentícias (BIDEAU; LORANGER; DANEAULT, 2018) e remoção de contaminantes aquáticos (TSHIKOVHI; MISHRA; MISHRA, 2020). Na área farmacêutica, pesquisas recentes têm demonstrado resultados promissores em ensaios de atividade antibacteriana, antifúngica (ZHANG et al., 2019) e no desenvolvimento de nanocarreadores magnéticos (SUPRAMANIAM et al., 2018). Liu e seus colaboradores (2015), utilizaram nanocelulose para remoção de íons $\mathrm{Cu}^{2+} \mathrm{e} \mathrm{Fe}^{3+}$, onde foi possível observar que o nanomaterial demonstra-se um excelente candidato na remoção de metais pesados.

As nanopartículas magnéticas destacam-se pelas características singulares que as tornam promissoras em estudos de adsorção e de atividade biológica (AL-JABARI et al., 2019), em especial, as nanopartículas com propriedades superparamagnéticas como a ferrita $\left(\mathrm{Fe}_{3} \mathrm{O}_{4}\right)$, respondem de forma rápida e forte a um campo magnético, além disso, apresentam alta biocompatibilidade, podendo ser utilizadas na modificação de superfícies de biopolímeros (SURESHKUMAR et al., 2016).

Neste sentido, o objetivo deste trabalho foi obter a celulose nanocristalina magnética (CNC. $\mathrm{Fe}_{3} \mathrm{O}_{4}$ ) com diferentes quantidades de $\mathrm{Fe}_{3} \mathrm{O}_{4}$ incorporada para posteriormente investigar a aplicação em diferentes áreas, sejam estas tecnológicas ou biológicas. 


\section{MATERIAL E MÉTODOS}

\section{OBTENÇÃO DE NANOCELULOSE MAGNÉTICA}

A magnetização da nanocelulose foi realizada conforme descrito por Rhoden et al. (2017). Para estas reações empregou-se um balão de fundo redondo de $250 \mathrm{~mL}$, contendo $100 \mathrm{~mL}$ de água ultrapura previamente desoxigenada, em seguida, adicionou-se $100 \mathrm{mg}$ de nanocelulose (Delaware $\left.{ }^{\circledR}\right)$ e $(100,500$ ou $1000 \mathrm{mg})$ cloreto de ferro II $\left(\mathrm{FeCl}_{2}\right)\left(\right.$ Sigma-Aldrich $\left.^{\circledR}\right)$ para promover a produção de nanocelulose com diferentes quantidades de $\mathrm{Fe}_{3} \mathrm{O}_{4}$ e, hidróxido de amônio $\left(\right.$ Synth $\left.^{\circledR}\right)$ até atingir $\mathrm{pH}$ oxidante $(\mathrm{pH} \approx 9,0)$. Após, a mistura foi submetida a radiação ultrassônica $\left(\mathrm{Elma}^{\circledR}\right.$, potência de $\left.150 \mathrm{~W}\right)$ e aquecimento a $80{ }^{\circ} \mathrm{C}$, intercalando as etapas, durante 60 minutos. Sequencialmente, a solução foi vertida para um béquer, e, com o auxílio de um ímã, o sólido foi lavado consecutivamente com metanol e acetona. Posteriormente, o material foi seco em estufa a $50{ }^{\circ} \mathrm{C}$ para total evaporação dos solventes.

\section{CARACTERIZAÇÕES}

\section{ESPECTROSCOPIA DE INFRAVERMELHO}

A espectroscopia de infravermelho é uma ferramenta importante e amplamente utilizada, pois fornece evidências da presença de grupos funcionais na estrutura orgânica. O infravermelho médio compreende números de onda entre $4000-400 \mathrm{~cm}^{-1}$, região de maior utilidade para caracterizar as estruturas contendo átomos de carbono.

O equipamento utilizado para caracterizar as amostras sintetizadas neste trabalho foi FTIR da Perkin-Elmer, modelo Spectro One. As pastilhas foram obtidas com $2 \mathrm{mg}$ de amostra e $200 \mathrm{mg}$ do suporte (KBr). O espectro aparece, em forma de bandas, resultante das vibrações das moléculas ao absorverem a radiação infravermelha (RHODEN, 2018).

\section{DIFRATOGRAMA DE RAIOS-X}

Outra técnica instrumental de análise utilizada na caracterização da nanocelulose é a difração de raios X. Métodos de difração medem diretamente a distância entre os planos paralelos de pontos do retículo cristalino. Esta informação é usada para determinar os parâmetros do reticulado de um cristal. Os métodos de difração também medem os ângulos entre os planos do reticulado. As análises de difração de raios-X foram realizadas utilizando o difratômetro da marca Bruker, modelo D2 Phaser, na faixa 
de $10^{\circ}$ a $60^{\circ}$ com velocidade de $0,0506426^{\circ}$ por segundo. As amostras foram maceradas e dispostas no porta-amostras de forma que ficassem com a superfície mais lisa possível (RHODEN, 2018). O tamanho médio do cristalito (D) é relacionado com a largura da meia altura dos picos difratados e o parâmetro de malha associado a posição dos picos, é dado pela equação (1) de Scherrer (1939):

$$
D_{h k l}=\frac{K \lambda}{\beta \cos \theta}
$$

Onde D é o tamanho médio do cristalito, $\mathrm{K}(\mathrm{K}=0,9)$ é a constante que depende da forma das partículas, $\chi(\chi=1,5418 \AA)$ o comprimento de onda da radiação eletromagnética, $\theta$ o ângulo de difração e $\beta$ a largura da meia altura do pico (FWHM). O valor de $\theta$ utilizado foi de $2 \theta \approx 35^{\circ}$ para a ferrita e $2 \theta \approx 22^{\circ}$ para todas as celuloses. O grau da cristalinidade das amostras foi calculado usando o método reportado Navarro-Pardo et al. (2013) na equação (2), onde Onde Ac é a área dos picos cristalinos e Aa é a área das cavidades amorfas das amostras.

$$
\% C=\frac{A c}{A c+A a} \times 100
$$

\section{RESULTADOS}

Para a obtenção da nanocelulose magnética, empregou-se a nanocelulose e o $\mathrm{FeCl}_{2}$ e posteriormente, submeteu-se a condições de oxidação. A figura 1 apresenta as condições do processo de magnetização do bionanopolímero, bem como o produto gerado pela reação.

Figura 1 - Estrutura química da nanocelulose magnética obtida no trabalho.

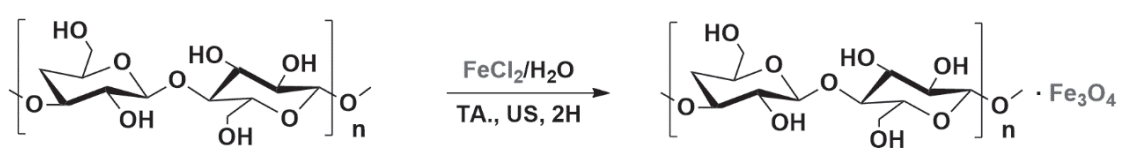

Fonte: construção do Autor

Na tabela 1 estão descritas as condições experimentais deste trabalho, além do rendimento das magnetizações.

Tabela 1 - Condições experimentais e rendimento das magnetizações.

\begin{tabular}{cccccccc}
\hline Reação & $\begin{array}{c}\mathbf{C N C} \\
(\mathbf{1 6 2} \mathbf{~ g / ~} \mathbf{~ m o l})\end{array}$ & $\begin{array}{c}\mathbf{F e C l}_{\mathbf{2}} \\
(\mathbf{1 2 7} \mathbf{~} / \mathbf{m o l})\end{array}$ & $\begin{array}{c}\mathbf{F e}_{\mathbf{3}} \mathbf{O}_{\mathbf{4}}{ }^{*} \\
\mathbf{( 2 3 2} \mathbf{~ g} \mathbf{m o l})\end{array}$ & $\begin{array}{c}\text { Tempo } \\
\text { (minutos) }\end{array}$ & Base & Rend. (mg) & Rend. (\%) \\
$\mathbf{R} 1$ & $100 \mathrm{mg}$ & $100 \mathrm{mg}$ & $54 \mathrm{mg}$ & 60 & $\mathrm{NH}_{4} \mathrm{OH}$ & $129 \mathrm{mg}$ & $83 \%$ \\
$\mathbf{R} 2$ & $100 \mathrm{mg}$ & $500 \mathrm{mg}$ & $270 \mathrm{mg}$ & 60 & $\mathrm{NH}_{4} \mathrm{OH}$ & $278 \mathrm{mg}$ & $75 \%$ \\
$\mathbf{R 3}$ & $100 \mathrm{mg}$ & $1000 \mathrm{mg}$ & $540 \mathrm{mg}$ & 60 & $\mathrm{NH}_{4} \mathrm{OH}$ & $493 \mathrm{mg}$ & $77 \%$ \\
\hline $\mathbf{R} 4$ & - & $800 \mathrm{mg}$ & $432 \mathrm{mg}$ & 60 & $\mathrm{NH}_{4} \mathrm{OH}$ & $249 \mathrm{mg}$ & $57,6 \%$ \\
\hline \multicolumn{7}{c}{ *Quantidade correspondente de $\mathrm{Fe}_{3} \mathrm{O}_{4}$ gerada a partir do $\mathrm{FeCl}_{2}$} \\
\end{tabular}


Conforme pode ser verificado nesta tabela, após a magnetização da $\mathrm{CNC}$ com $\mathrm{FeCl}_{2}$ na proporção 1:1 (R1), obteve-se 129 mg em massa do material, o produto da reação de magnetização 1:5 (R2), rendeu 278 mg, na reação 1:10 (R3) obteve-se a formação de $493 \mathrm{mg}$, enquanto a síntese de $\mathrm{Fe}_{3} \mathrm{O}_{4}(\mathbf{R} 4)$, produziu, em massa de, $249 \mathrm{mg}$ da nanopartícula magnética. Estes resultados se mostram congruentes aos encontrados por Rhoden (2018) ao magnetizar o óxido de grafeno, partindo-se de metodologia semelhante. A metodologia empregada na incorporação de ferrita à CNC, mostrou-se altamente eficiente, com bons rendimentos, baixa necessidade energética e redução do tempo de processo, comparado aos métodos de co-precipitação, microemulsão, deposição termal existentes na literatura, que utilizam altas temperaturas, grandes quantidades de $\mathrm{FeCl}_{2}$ e, ainda um elevado tempo de sonicação e de aquecimento. Ressalta-se, entretanto, que atualmente a literatura não reporta resultados referentes a rendimentos na incorporação de nanopartículas magnéticas (ABU-DIEF; ABDEL-FATAH, 2017; MAITY; DING; XUE, 2008).

\section{ESPECTROSCOPIA DE INFRAVERMELHO}

Na figura abaixo são apresentados os resultados de espectroscopia de infravermelho da ferrita, nanocelulose e nanoceluloses magnéticas com diferentes proporções de ferrita incorporada.

Figura 2 - Espectro de infravermelho da nanocelulose e nanoceluloses magnéticas.

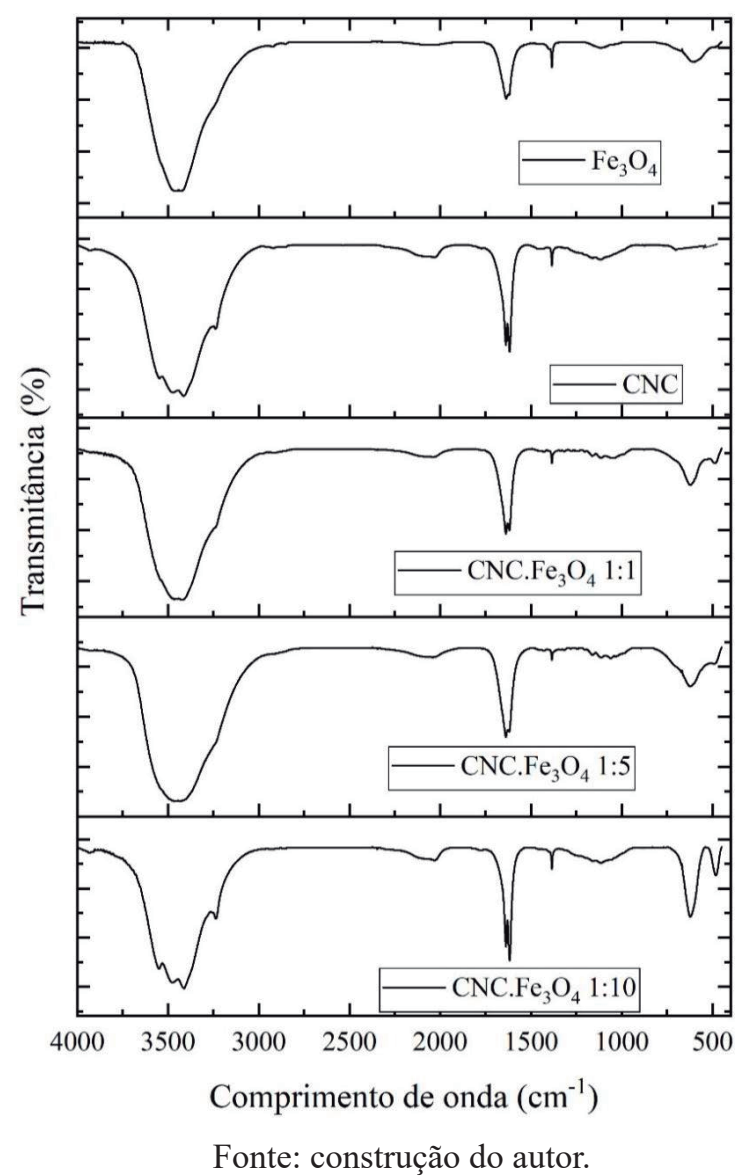


Os picos de absorção da nanocelulose são observados na região de $3400 \mathrm{~cm}^{-1}$, representando vibrações do alongamento da ligação $\mathrm{O}-\mathrm{H}$, em torno de $1600 \mathrm{~cm}^{-1}$, uma banda indicando a absorção de água pelas moléculas da celulose. Além disso, na região de $1400 \mathrm{~cm}^{-1}$ atribuída a um $\mathrm{CH}_{2}$ simétrico, próximo a $1100 \mathrm{~cm}^{-1}$ representando alongamentos $\mathrm{C}-\mathrm{C}$ e $\mathrm{C}-\mathrm{O}$, e em $1050 \mathrm{~cm}^{-1}$ o anel piranose $\mathrm{C}_{\text {alquil }} \mathrm{O}$, relacionado às vibrações de éter e ligações $\beta$-glicosídicas (AGUAYO et al., 2018; ADEL et al., 2011).

Além dos picos constantes, nos FTIR das nanoceluloses magnéticas, observou-se o aparecimento de uma banda inexistente anteriormente na faixa de $600 \mathrm{~cm}^{-1}$ pelas vibrações de estiramento de $\mathrm{Fe}-\mathrm{O}$, indicando a presença de $\mathrm{Fe}_{3} \mathrm{O}_{4}$ incorporado à CNC (LOPEZ et al., 2010; RHODEN et al., 2017). Observa-se ainda a intensificação da banda na faixa de $600 \mathrm{~cm}^{-1}$ e medida em a quantidade de $\mathrm{Fe}_{3} \mathrm{O}_{4}$ incorporada ao biopolímero aumenta.

\section{DIFRATOGRAMA DE RAIOS-X}

Na tabela 2, estão representados os percentuais da cristalinidade e o tamanho médio do cristalito, calculados a partir das equações 1 e 2 .

Tabela 2 - Grau de cristalinidade das amostras e tamanho médio do cristalito.

\begin{tabular}{ccc}
\hline Amostra & Grau de cristalinidade (\%) & Tamanho médio do cristalito (nm) \\
\hline $\mathrm{CNC}$ & 99,99 & 44,48 \\
\hline $\mathrm{CNC} . \mathrm{Fe}_{3} \mathrm{O}_{4} 1: 1$ & 82,10 & 20,28 \\
\hline $\mathrm{CNC} \cdot \mathrm{Fe}_{3} \mathrm{O}_{4} 1: 5$ & 78,82 & 20,53 \\
\hline $\mathrm{CNC} . \mathrm{Fe}_{3} \mathrm{O}_{4} 1: 10$ & 39,05 & 23,54 \\
$\mathrm{Fe}_{3} \mathrm{O}_{4}$ & 50,96 & 3,11 \\
\hline
\end{tabular}

De acordo com os dados obtidos experimentalmente, observa-se que o grau de cristalinidade da nanocelulose diminuiu à medida que o incremento de $\mathrm{Fe}_{3} \mathrm{O}_{4}$ na sua estrutura aumentou, tal fato explica-se pelas etapas de aquecimento e sonicação, empregados na magnetização dos bionanopolímeros (SALMI et al., 2019; SZYMAŃSKA-CHARGO et al., 2018). Em contrapartida, o tamanho médio do cristalito de todas as CNC magnéticas diminuiu com a adição de ferrita. Meios altamente oxidantes, contendo metais de transição, como o $\mathrm{Fe}^{2+}$ na presença de uma base, aumentam a taxa de hidrólise de materiais e, consequentemente diminuem o tamanho da partícula (ISLAM et al., 2014).

As CNC magnéticas demonstraram tamanho de partícula semelhante ao encontrado por Khalilzadeh et al. (2020) que desenvolveram um nanocompósito magnético dopado com cobre com tamanho médio de $25 \mathrm{~nm}$, também calculado por meio da equação de Scherrer. As nanopartículas de $\mathrm{Fe}_{3} \mathrm{O}_{4}$ sintetizadas tiveram tamanho médio de 3,11 nm, metodologias de co-precipitação empregando somente cloreto de ferro II e temperaturas próximas a $80{ }^{\circ} \mathrm{C}$, reportam valores em torno de $7 \mathrm{~nm}$, corroborando com os resultados deste trabalho (SARAGI et al., 2018). Na figura 3 são apresentados 
os difratogramas de raios-X (DRX) da ferrita, celulose nanocristalina e celulose nanocristalina decorada com diferentes proporções de ferrita.

Figura 3 - Difração de raios-X da celulose nanocristalina e das celuloses nanocristalinas magnéticas.

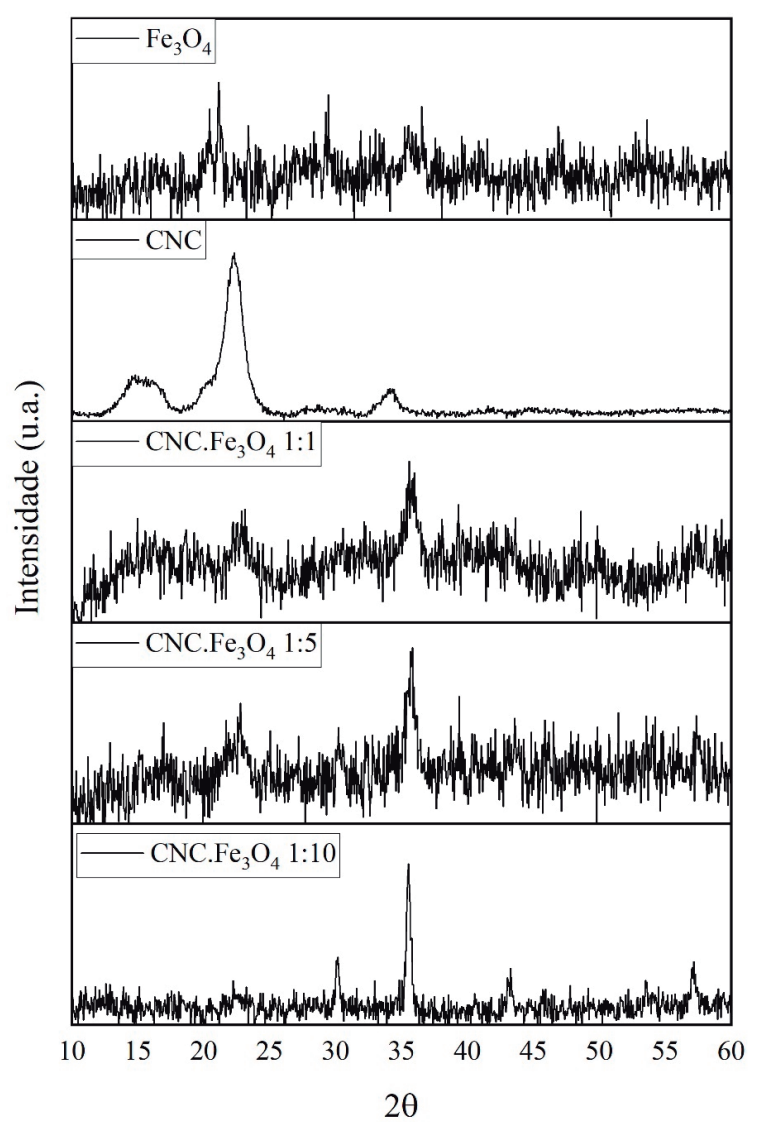

Fonte: construção do autor.

Em $2 \theta \approx 22^{\circ}$ é possível observar um pico cristalino característico da $\mathrm{CNC}$, em $2 \theta \approx 14^{\circ}, 34^{\circ}$ indicando a presença dos picos amorfos em sua estrutura, corroborando com os resultados encontrados por Trifol e colaboradores (2016). A presença dos picos referentes ao $\mathrm{Fe}_{3} \mathrm{O}_{4}$ podem ser observados em $2 \theta \approx 36^{\circ}, 45^{\circ}$ e $56^{\circ}$ (SHAGHOLANI; GHOREISHI; MOUSAZADEH, 2015; RHODEN et al., 2017), observa-se também que, o pico em torno de $2 \theta \approx 22^{\circ}$ da nanocelulose empregada nas reações de magnetização foi parcialmente suprimido pela incorporação de ferrita a sua estrutura (YANG et al., 2016). No entanto, cabe ressaltar que o fato do alguns difratogramas de raios-X apresentarem um alto ruído, pode ser explicado pela presença de nanopartículas magnéticas, que por sua vez, possuem uma estrutura cristalina (UNTERWEGER et al., 2015).

\section{CONCLUSÃO}

A partir dos resultados obtidos, foi possível concluir que houve a incorporação de nanopartículas magnéticas nas nanoceluloses. As técnicas instrumentais de difração de raios-X e espectroscopia de 
infravermelho, foram apropriadas para a confirmação da magnetização, assim como a resposta positiva pela aproximação de um campo magnético. Além disso, verificou-se que as condições experimentais empregadas nas reações de magnetização, influenciaram em parâmetros importantes como no tamanho de partícula e o grau de cristalinidade das amostras. Esta proposta para a magnetização é de rápida e de fácil obtenção. No entanto, estudos subsequentes são necessários quanto a toxicidade destes nanomateriais, de suma importância para a aplicação em sistemas biológicos.

\section{AGRADECIMENTOS}

Os autores agradecem a FAPERGS e ao CNPq e a CAPES e a Universidade Franciscana UFN pelas Bolsas concedidas.

\section{REFERÊNCIAS}

ABU-DIEF, A. M.; ABDEL-FATAH, S. M. Development and functionalization of magnetic nanoparticles as powerful and green catalysts for organic synthesis. Beni-Suef University Journal of Basic and Applied Sciences, v. 7, n. 1, p. 55-67, 2017.

ADEL, A. M. et al. Characterization of microcrystalline cellulose prepared from lignocellulosic materials. Part II: Physicochemical properties. Carbohydrate Polymers, v. 83, n. 2, p. 676-687, 2011.

AGUAYO, M. G. et al. Isolation and characterization of cellulose nanocrystals from rejected fibers originated in the kraft pulping process. Polymers, v. 10, n. 10, p. 1145, 2018.

AL-JABARI, M. H. et al. Adsorption study of levofloxacin on reusable magnetic nanoparticles: Kinetics and antibacterial activity. Journal of Molecular Liquids, v. 291, p. 111249, 2019.

BIDEAU, B.; LORANGER, E.; DANEAULT, C. Nanocellulose-polypyrrole-coated paperboard for food packaging application. Progress in Organic Coatings, v. 123, p. 128-133, 2018.

ISLAM, M. T. et al. Preparation of nanocellulose: A review. AATCC Journal of Research, v. 1, n. 5, p. 17-23, 2014.

KHALILZADEH, M. A. et al. Green synthesis of magnetic nanocomposite with iron oxide deposited on cellulose nanocrystals with copper $\left(\mathrm{Fe}_{3} \mathrm{O}_{4} @ \mathrm{CNC} / \mathrm{Cu}\right)$ : investigation of catalytic activity for the development of a venlafaxine electrochemical sensor. Industrial \& Engineering Chemistry Research, v. 59, n. 10, p. 4219-4228, 2020. 
LEFATSHE, K.; MUIVA, C. M.; KEBAABETSWE, L. P. Extraction of nanocellulose and in-situ casting of $\mathrm{ZnO} /$ cellulose nanocomposite with enhanced photocatalytic and antibacterial activity. Carbohydrate polymers, v. 164, p. 301-308, 2017.

LIN, Y. J.; SHATKIN, J. A.; KONG, F. Evaluating mucoadhesion properties of three types of nanocellulose in the gastrointestinal tract in vitro and ex vivo. Carbohydrate polymers, v. 210, p. 157-166, 2019.

LIU, P. et al. Nanocelluloses and their phosphorylated derivatives for selective adsorption of Ag+, $\mathrm{Cu} 2+$ and $\mathrm{Fe} 3+$ from industrial effluents. Journal of hazardous materials, v. 294, p. 177-185, 2015.

LOPEZ, J. A. et al. Synthesis and characterization of $\mathrm{Fe}_{3} \mathrm{O}_{4}$ magnetic nanofluid. Revista Latinoamericana de Metalurgia y Materiales, v. 30, n. 1, p. 60-66, 2010.

MAITY, D.; DING, J. U. N.; XUE, Jun-Min. Synthesis of magnetite nanoparticles by thermal decomposition: time, temperature, surfactant and solvent effects. Functional Materials Letters, v. 1, n. 03, p. 189-193, 2008.

NAVARRO-PARDO, F. et al. Effects on the thermo-mechanical and crystallinity properties of nylon 6, 6 electrospun fibres reinforced with one dimensional (1D) and two dimensional (2D) carbon. Materials, v. 6, n. 8 , p. 3494-3513, 2013.

PATTERSON, A. L. The Scherrer formula for X-ray particle size determination. Physical review, v. 56, n. 10, p. 978-982, 1939.

RHODEN, C. R. B. et al. Síntese fácil e direta do óxido de grafeno magnético. Disciplinarum Scientia | Naturais e Tecnológicas, v. 18, n. 2, p. 149-147, 2017.

RHODEN, C. R. B. Síntese de análogos de peptídeos incorporados a materiais nanoparticulados de carbono funcionalizados via reações multicomponentes. 2018. 107f. Tese de Doutorado (Doutor em Nanociências), Universidade Franciscana, 2018.

RIBEIRO, R. S. A. et al. Production of nanocellulose by enzymatic hydrolysis: Trends and challenges. Engineering in Life Sciences, v. 19, n. 4, p. 279-291, 2019.

SALIMI, S. et al. Production of nanocellulose and its applications in drug delivery: A critical review. ACS Sustainable Chemistry \& Engineering, v. 7, n. 19, p. 15800-15827, 2019. 
SHAGHOLANI, H.; GHOREISHI, S. M.; MOUSAZADEH, M. Improvement of interaction between PVA and chitosan via magnetite nanoparticles for drug delivery application. International journal of biological macromolecules, v. 78, p. 130-136, 2015.

SARAGI, T. et al. The impact of synthesis temperature on magnetite nanoparticles size synthesized by co-precipitation method. JPhCS, v. 1013, n. 1, p. 012190, 2018.

STANISŁAWSKA, A. Bacterial nanocellulose as a microbiological derived nanomaterial. Advances in materials science, v. 16, n. 4, p. 45-57, 2016.

SZYMAŃSKA-CHARGOT, M. et al. Effect of ultrasonication on physicochemical properties of apple based nanocellulose-calcium carbonate composites. Cellulose, v. 25, n. 8, p. 4603-4621, 2018.

SUPRAMANIAM, J. et al. Magnetic nanocellulose alginate hydrogel beads as potential drug delivery system. International journal of biological macromolecules, v. 118, p. 640-648, 2018.

SURESHKUMAR, V. et al. Fabrication of chitosan-magnetite nanocomposite strip for chromium removal. Applied Nanoscience, v. 6, n. 2, p. 277-285, 2016.

TAN, Hoi-Fang.; OOI, B. S.; LEO, C. P. Future perspectives of nanocellulose-based membrane for water treatment. Journal of Water Process Engineering, v. 37, p. 101502, 2020.

THOMAS, P. et al. Comprehensive review on nanocellulose: Recent developments, challenges and future prospects. Journal of the Mechanical Behavior of Biomedical Materials, p. 103884, 2020.

TRIFOL, J. et al. A comparison of partially acetylated nanocellulose, nanocrystalline cellulose, and nanoclay as fillers for high-performance polylactide nanocomposites. Journal of Applied Polymer Science, v. 133, n. 14, 2016.

TSHIKOVHI, A.; MISHRA, S. B.; MISHRA, A. K. Nanocellulose-based composites for the removal of contaminants from wastewater. International Journal of Biological Macromolecules, 2020.

UNTERWEGER, H. et al. Hypericin-bearing magnetic iron oxide nanoparticles for selective drug delivery in photodynamic therapy. International journal of nanomedicine, v. 10, p. 6985, 2015. 
VIANA, A. R., et al. Cytotoxicity study of graphene oxide against vero lineage cells. Disciplinarum Scientia Naturais e Tecnológicas, v. 20, n. 3, p. 355-364, 2019.

YANG, S. et al. Preparation of graphene oxide decorated $\mathrm{Fe}_{3} \mathrm{O}_{4} @ \mathrm{SiO}_{2}$ nanocomposites with superior adsorption capacity and SERS detection for organic dyes. Journal of Nanomaterials, v. 16, n. 1, p. $337-345,2015$.

ZAMAN, A. et al. Preparation, Properties, and Applications of Natural Cellulosic Aerogels: A Review. Energy and Built Environment, v. 1, n. 1, p. 60-76, 2020.

ZHANG, X.; et al. Hydrothermal synthesis of Ag nanoparticles on the nanocellulose and their antibacterial study. Inorganic Chemistry Communications, v. 100, p. 44-50, 2019.

ZINGE, C.; KANDASUBRAMANIAN, B. Nanocellulose based Biodegradable Polymers. European Polymer Journal, p. 109758, 2020. 
